\title{
Iron overload contributes to general anaesthesia-induced neurotoxicity and cognitive deficits
}

\author{
Jing Wu', Jian-Jun Yang ${ }^{2}$, Yan Cao ${ }^{1}$, Huihui Li ${ }^{1}$, Hongting Zhao ${ }^{1}$, Shuofei Yang ${ }^{3^{*}}$ and Kuanyu Li $^{{ }^{*}}$
}

\begin{abstract}
Background: Increasing evidence suggests that multiple or long-time exposure to general anaesthesia (GA) could be detrimental to cognitive development in young subjects and might also contribute to accelerated neurodegeneration in the elderly. Iron is essential for normal neuronal function, and excess iron in the brain is implicated in several neurodegenerative diseases. However, the role of iron in GA-induced neurotoxicity and cognitive deficits remains elusive.
\end{abstract}

Methods: We used the primary hippocampal neurons and rodents including young rats and aged mice to examine whether GA impacted iron metabolism and whether the impact contributed to neuronal outcomes. In addition, a pharmacological suppression of iron metabolism was performed to explore the molecular mechanism underlying GA-mediated iron overload in the brain.

Results: Our results demonstrated that GA, induced by intravenous ketamine or inhalational sevoflurane, disturbed iron homeostasis and caused iron overload in both in vitro hippocampal neuron culture and in vivo hippocampus. Interestingly, ketamine- or sevoflurane-induced cognitive deficits, very likely, resulted from a novel iron-dependent regulated cell death, ferroptosis. Notably, iron chelator deferiprone attenuated the GA-induced mitochondrial dysfunction, ferroptosis, and further cognitive deficits. Moreover, we found that GA-induced iron overload was activated by NMDAR-RASD1 signalling via DMT1 action in the brain.

Conclusion: We conclude that disturbed iron metabolism may be involved in the pathogenesis of GA-induced neurotoxicity and cognitive deficits. Our study provides new vision for consideration in GA-associated neurological disorders.

Keywords: Iron, Ferroptosis, General anaesthesia, Neurotoxicity, Cognition

\section{Introduction}

Millions of paediatric and elderly patients undergoing surgery require general anaesthesia (GA) each year worldwide. Accumulating evidence is forcing the anaesthesia community to question the safety of GA at the extremes of age $[1,2]$. A large body of preclinical as well

\footnotetext{
*Correspondence: doctor_yangshuofei@163.com; likuanyu@nju.edu.cn ${ }^{3}$ Department of Vascular Surgery, Renji Hospital, School of Medicine, Shanghai Jiaotong University, Shanghai 200127, China

'Jiangsu Key Laboratory of Molecular Medicine, Medical School of Nanjing University, 22 Hankou Road, Nanjing 210093, China

Full list of author information is available at the end of the article
}

as some retrospective clinical studies suggest that general anaesthetics (GAs) can have profound and longlasting effects in young subjects by increasing neuronal death and reducing neurogenesis [3]. On the other hand, elderly patients are recognized to take an increased risk of postoperative cognitive dysfunction (POCD) after GA and surgery [4]. Although GAs have been used clinically for over a century, both their targets of action and the nature of any potentially neurotoxic side effects remain incompletely understood.

(c) The Author(s). 2020 Open Access This article is licensed under a Creative Commons Attribution 4.0 International License, which permits use, sharing, adaptation, distribution and reproduction in any medium or format, as long as you give appropriate credit to the original author(s) and the source, provide a link to the Creative Commons licence, and indicate if changes were made. The images or other third party material in this article are included in the article's Creative Commons licence, unless indicated otherwise in a credit line to the material. If material is not included in the article's Creative Commons licence and your intended use is not permitted by statutory regulation or exceeds the permitted use, you will need to obtain permission directly from the copyright holder. To view a copy of this licence, visit http://creativecommons.org/licenses/by/4.0/ The Creative Commons Public Domain Dedication waiver (http://creativecommons.org/publicdomain/zero/1.0/) applies to the data made available in this article, unless otherwise stated in a credit line to the data. 
GAs can be broadly classified as either volatile inhaled compounds (such as nitrous oxide, sevoflurane, isoflurane, and desflurane) or intravenously administered compounds (such as propofol and ketamine). Two main targets have been extensively described to the GAs: the excitatory $\mathrm{N}$-methyl-D-aspartate (NMDA) glutamate receptors and the inhibitory gamma-aminobutyric acid $\left(\mathrm{GABA}_{\mathrm{A}}\right)$ receptors. By binding glutamate, NMDA receptors (NMDAR) gate an influx of $\mathrm{Ca}^{2+}$ and $\mathrm{Na}^{+}$to cause membrane depolarization, while GABA binds to $\mathrm{GABA}_{\mathrm{A}}$ receptors, an influx of $\mathrm{Cl}^{-}$results in hyperpolarization [5]. Most GAs, such as ketamine and sevoflurane, activate the inhibitory ion channels and/or inhibit the excitatory ion channels and thereby reduce neuronal excitability and lead to GA [5]. There are other potential targets of anaesthetic relevance including glycine receptors and $\mathrm{K}^{+}$channels $[6,7]$. This diversity of potential targets increases the probability of both positive and negative non-anaesthetic effects. NMDA neurotoxicity can be mediated through an iron transport signalling cascade, i.e., NMDAR upregulation might promote iron overload by enhancing DMT1 (divalent metal transporter 1)-mediated iron influx [8], stimulating iron releasing from lysosome [9], thus causing iron-induced cell damage. Therefore, we question whether iron is involved in GA-induced neurotoxicity.

Iron is one of the most abundant trace elements in the human body. Iron plays a critical role in brain development and physiology, neurotransmitter synthesis (including monoamine transmitters and GABA), cytoplasmic protein function, and mitochondrial reactions [10]. Nontransferrin-bound iron (NTBI) in the central nervous system appears to be the main physiologically available iron source, and DMT1 is a very important iron transporter for iron uptake [11, 12]. Excess iron in the brain causes neurotoxicity and significant cognitive impairments, which has been implicated in the pathogenesis of several neurological disorders including hypoxic ischemic brain injury and periventricular white matter injury in neonates [13] and neurodegenerative disorders in elders [10, 14]. Ferroptosis is an iron-dependent regulated cell death process that was discovered recently and is usually accompanied by lipid peroxidation during the cell death. It is closely related to the pathophysiological processes of many diseases [15]. A recent study has shown in vitro that pretreatment with the selective ferroptosis inhibitor, ferrostatin-1, preserved mitochondrial function and mitigated neuronal cell death secondary to isoflurane exposure, suggesting that ferroptosis contributes to isoflurane neurotoxicity [16]. Thus, we propose that disruption of iron homeostasis and iron-dependent ferroptosis contribute GA-induced neurotoxicity.

In this study, we examined the effects of intravenous ketamine and inhalational sevoflurane on brain iron homeostasis, mitochondrial function, and neuronal outcomes in vitro and in vivo. Our hypothesis was confirmed by the findings that GA-induced iron overload and iron chelation by deferiprone (DFP) reversed the neurotoxicity. Moreover, we found that GA-induced neurotoxicity was regulated by NMDAR-RASD1 signalling through DMT1 upregulation.

\section{Materials and methods}

\section{Animals}

Sprague-Dawley rat pups at postnatal day (PND) 6 and 15 -month-old male C57BL/6 mice were used in the present study. All experimental procedures and protocols were reviewed and approved by the Animal Investigation Ethics Committee of Nanjing University and were performed in accordance with the Guidelines for the Care and Use of Laboratory Animals from the National Institutes of Health, USA. The animals were housed in a room maintained under standard environmental condition (temperature $22-24^{\circ} \mathrm{C}$, a $12 \mathrm{~h} \mathrm{light/dark}$ cycle, and $50 \pm 10 \%$ humidity) with free access to food and water. The rat pups were housed with their mothers till PND 20. At PND 21, the pups were weaned and housed 4-5 per cage in standard condition.

\section{General anaesthesia}

GA was performed based on our previous optimization $[17,18]$, in which ketamine $(75 \mathrm{mg} / \mathrm{kg})$ intraperitoneally or $3 \%$ sevoflurane $2 \mathrm{~h}$ inhalation daily for three consecutive days can induce neurotoxicity and cognitive impairments. For ketamine GA, rat pups at PND 6 or 15 -month-old mice received ketamine $(75 \mathrm{mg} / \mathrm{kg})$ intraperitoneally daily for three consecutive days. For sevoflurane GA, animals were put in an anaesthetizing chamber delivered with $3 \%$ sevoflurane plus $30 \%$ oxygen $\left(\mathrm{O}_{2}\right)$ for $2 \mathrm{~h}$ daily for three consecutive days. For control experiments, $30 \% \mathrm{O}_{2}$ was delivered at the same flow rate. For drug treatment, DFP $(75 \mathrm{mg} / \mathrm{kg}$, intraperitoneally, synthesized in China Peptides Co., Ltd., Shanghai, China) or DMT1i $(50 \mathrm{mg} / \mathrm{kg}$, orally, MedChemExpress, China) $[19,20]$ was administered to the animals $1 \mathrm{~h}$ before GA daily for three consecutive days.

The composition of the chamber gas was continuously monitored using a DatexTM infrared analyzer (Capnomac, Helsinki, Finland). Animals were kept normothermic throughout the experiment. Animals were kept warm on a plate heated to $37 \pm 1{ }^{\circ} \mathrm{C}$ and returned to the cage until recovery of the right reflex after GA exposure.

\section{Rat hippocampal neuronal culture and anaesthetic exposure}

Primary neuronal cultures were prepared from embryonic day 16-17 (E16-17) embryos of Sprague-Dawley rats as previously described [21]. Neurons were dissociated and 
seeded on Poly-Lysine pretreated culture dishes with neurobasal medium, supplemented with B-27, glutamine, antibiotics, and 5\% FBS (Invitrogen GIBCO Life Technologies, Carlsbad, CA, USA). After $2 \mathrm{~h}$ incubation, primary cultures were maintained in neurobasal medium without $\mathrm{FBS}$ in $5 \% \mathrm{CO}_{2}$ incubator at $37^{\circ} \mathrm{C}$. Half of the culture medium was refreshed every 3 days. After 9 days of culture (DIV 9), the neurons were treated with ketamine $(10 \mu \mathrm{M})$ in $5 \% \mathrm{CO}_{2}$ incubator or $3 \%$ sevoflurane plus $5 \%$ $\mathrm{CO}_{2}$ for $6 \mathrm{~h}$ at $37^{\circ} \mathrm{C}$, whereas the control group was maintained in same amount of culture medium. For drug treatment, DFP $(100 \mu \mathrm{M})$ or DMT1i $(3 \mu \mathrm{M})$ was added to the culture medium 30 min before GA exposure.

\section{Cell viability assays}

The neurons were seeded in 96-well plates for 9 days. Cell Counting Kit-8 (Beyotime Biotechnology) was used to assess cell viability according to the manufacturer's instructions. Results were expressed as the percentage of reduction of absorbance at $450 \mathrm{~nm}$ by calibration with the absorbance of the control cells.

\section{Lactate dehydrogenase (LDH) assays}

$\mathrm{LDH}$ is a stable cytoplasmic enzyme present in all types of cells and released into the cell culture medium through damaged plasma membrane. The neuron cells were plated into 12-well plates for 9 days, and LDH release was measured by LDH Assay Kit according to the manufacturer's instructions (Sigma-Aldrich, USA) following the treatment as needed.

\section{Mitochondria isolation}

Mitochondria from the hippocampus or primary hippocampal neurons were isolated using Qproteome Mitochondria isolation kit (Qiagen, Cat. No. 37612, USA). In brief, tissue or cells were homogenized in ice-chilled Dounce homogenizers using isolation buffer and centrifuged at $1000 \mathrm{~g}$ for $10 \mathrm{~min}$ at $4{ }^{\circ} \mathrm{C}$. The supernatant was then centrifuged at $6000 \mathrm{~g}$ for $10 \mathrm{~min}$ to separate the mitochondria and cytoplasm. The later mitochondriaenriched pellets were gently resuspended and washed with isolation buffer, then pelleted by centrifugation at $6000 \mathrm{~g}$ for $10 \mathrm{~min}$. The later supernatant was transferred into new EP tubes and centrifuged at $12,000 \mathrm{~g}$ for $10 \mathrm{~min}$ at $4{ }^{\circ} \mathrm{C}$. This supernatant was considered as cytosolic fraction. The protein was determined by the Micro BCA protein assay kit (Beyotime Institute of Biotechnology).

\section{Ferrozine iron assays}

Iron content was measured using a colorimetric ferrozine-based assay with some modifications [22]. Briefly, $22 \mu$ l concentrated $\mathrm{HCl}(11.6 \mathrm{~mol} / \mathrm{L})$ was added to $100 \mu \mathrm{l}$ cell lysate $(\sim 500 \mu \mathrm{g}$ total protein). The mixed sample was heated at $95^{\circ} \mathrm{C}$ for $20 \mathrm{~min}$, then centrifuged at $12,000 \mathrm{~g}$ for $10 \mathrm{~min}$. Supernatant was transferred very gently into fresh tubes. Ascorbate was added to reduce the $\mathrm{Fe}(\mathrm{III})$ into $\mathrm{Fe}(\mathrm{II})$. After 2 min of incubation at room temperature, ferrozine and saturate ammonium acetate (NH4Ac) were sequentially added to each tube and the absorbance was measured at $570 \mathrm{~nm}$ (BioTek EL x 800, Shanghai, China) within 30 min.

\section{Determination of mitochondrial swelling}

To initiate mitochondrial swelling by $\mathrm{Ca}^{2+}$ uptake, freshly isolated mitochondria were suspended in mitochondrial suspension buffer [ $120 \mathrm{mM} \mathrm{KCl}, 25 \mathrm{mM}$ sucrose, $5 \mathrm{mM}$ KH2PO4, $0.1 \mathrm{mM}$ EGTA, 20 MOPS (pH 7.2 )] in the presence of $5 \mathrm{mM}$ malate and $5 \mathrm{mM}$ pyruvate as substrates. Swelling was recorded as the decrease of the density of the mitochondrial matrix at $540 \mathrm{~nm}$ with a UV/Vis spectrophotometer after adding $0.5 \mathrm{mM}$ $\mathrm{Ca}^{2+}$ into the medium.

\section{Determination of mitochondrial membrane potential (MMP) levels and ATP contents}

Levels of MMP and ATP contents were determined in hippocampal neurons using a 5,5',6,6'-tetrachloro- $1,1^{\prime}$, $3,3^{\prime}$ tetraethylbenzimidazolylcarbocyanine iodide (JC-1) mitochondrial membrane potential detection kit (Solarbio Science \& Technology Co. Ltd.) and ATP content assay kit (Beyotime Biotech) according to the manufacturer's instructions.

\section{Mitochondrial morphology imaging}

Mitochondrial morphology was observed under the confocal microscopy following staining with the mitochondria targeting dye MitoTracker Red CMXRos (Beyotime Biotech). After GA treatment for $6 \mathrm{~h}$, the neurons were washed and stained with $20 \mathrm{nM}$ MitoTracker Red CMXRos in neurobasal medium for $30 \mathrm{~min}$ at $37^{\circ} \mathrm{C}$ in dark in a $5 \% \mathrm{CO}_{2}$ incubator. The cells were then washed with HBSS and immersed in neurobasal medium to prevent cell damage. Images were obtained with confocal microscopy (Fluoview FV 10i, Olympus) and analysed using FV10-ASW 2.1 Viewer software.

\section{Measurement of cellular ROS, MDA, and GSH levels}

Intracellular reactive oxygen species (ROS) levels were estimated using a fluorescence-labelled probe DCFH-DA (Beyotime Biotech). Cellular malondialdehyde (MDA) and glutathione (GSH) levels were measured following the manufacturer's instructions (Nanjing Jiancheng Bioengineering Institute, Nanjing, China).

\section{Western blot analysis}

Proteins were obtained from the hippocampal tissue or cultured neurons or isolated mitochondria. For western blotting, $35-50 \mu \mathrm{g}$ protein was added per lane of $12 \%$ 
SDS-PAGE. Primary antibodies were diluted in primary antibody dilution buffer (Beyotime Biotech., Shanghai, China). Antibodies used included the following: antiGAPDH (Abgent Biotech. Co. Ltd., Suzhou, China), anti-ferritin, superoxide dismutase 2 (SOD2), Mitoferrin1, Drp1, Mfn2, RASD1, NMDAR1, and NMDAR2A (Abcam, Cambridge, MA, USA), anti-TfR1 (Zymed, San Francisco, CA, USA), anti-IRP2 (polyclonal, raised from rabbit), and anti-DMT1 (Alpha Diagnostic International, San Antonio, TX, USA). Detection was performed using peroxidase-conjugated secondary antibodies (Thermo Fisher Scientific, Waltham, UK). Quantification of the density of the western bands was done with programme ImageJ (http://rsb.info.nih.gov/ij/).

\section{Morris water maze tests}

To investigate spatial learning and memory function, we subjected the rats ( $n=12$ for each group) to the Morris water maze (MWM) tests (XR-XM101; Shanghai Xinruan Information Technology Co., Ltd., Shanghai, China) at PND 60. The MWM was a black metal tank $(120 \mathrm{~cm}$ in diameter, $60 \mathrm{~cm}$ in depth) equipped with a platform $(10 \mathrm{~cm}$ in diameter) $1-2 \mathrm{~cm}$ below the surface of the water. The MWM task was performed according to our previous study [18]. Briefly, it consists of two phases, training phase for five consecutive days and probe trial phase on day 6 . In the training phase, the rat was allowed to face to the pool wall in four random places $(\mathrm{N}, \mathrm{S}, \mathrm{E}, \mathrm{W})$ in the pool to find the fixed platform. Release positions were randomly predetermined. The trial was terminated once the rat reached the platform. If the rat failed to reach the platform within $60 \mathrm{~s}$, it would be guided to the platform and allowed to stay for $15 \mathrm{~s}$, then the latency was recorded for $60 \mathrm{~s}$. In the probe test, a single-probe trial was conducted with the original platform removed $24 \mathrm{~h}$ after the last training session. The rat was released at a random start position and allowed to swim for $60 \mathrm{~s}$ in the pool.

\section{Fear conditioning tests}

To measure the abilities of learning and memory, we employed the fear conditioning paradigm $(30 \mathrm{~cm}$ long $\times$ $26 \mathrm{~cm}$ wide $\times 22 \mathrm{~cm}$ high, XR-XC404, Shanghai Softmaze Information Technology Co. Ltd.) to the aged mice $24 \mathrm{~h}$ after GA. Each mouse was exposed in the conditioning chamber for $3 \mathrm{~min}$ of accommodation then one tone-foot-shock pairing (tone, $30 \mathrm{~s}, 65 \mathrm{~dB}, 3 \mathrm{kHz}$; footshock, $3 \mathrm{~s}, 0.75 \mathrm{~mA}$ ) was delivered. The contextual fear conditioning test was performed $24 \mathrm{~h}$ later by placing mice back to the same test chamber for $5 \mathrm{~min}$ without any stimulation. Two hours later, each mouse was placed in a novel chamber altered in shape, colour, and smell, and the same tone was presented for $3 \mathrm{~min}$ without the foot shock to evaluate tone fear conditioning.
Cognitive deficits in the test were assessed by measuring the length of time of "freezing behaviour," which is defined as a completely immobile posture except for respiratory efforts. Freezing behaviour was automatically recorded by the video tracking system.

\section{Statistics analysis}

Data were presented as mean \pm SEM and analysed by the Statistical Product for Social Sciences (SPSS; version 18.0, IL, USA). The difference between the groups was determined by one-way analysis of variance followed by the Bonferroni test. Comparisons for the spatial training sessions of MWM were performed by repeated two-way ANOVA followed by the LSD test. A $p$ value $<0.05$ was considered statistically significant.

\section{Results}

\section{GA causes hippocampal iron overload}

Multiple or long-time exposure to GAs may produce profound and long-lasting effects in extremes of lifetime by reducing neurogenesis and/or accelerating neurodegeneration. Iron deposition in the hippocampus, cortical areas, and basal ganglia has been revealed to impair the spatial memory, aversive memory, and recognition memory [10]. To test our hypothesis that GA would induce iron overload, which could be involved in GA-induced neurotoxicity in the brain, we first measured the iron content in cultured hippocampal neurons and in the hippocampus of developing rats and aged mice after exposure to ketamine or sevoflurane. The results showed that both ketamine and sevoflurane caused cytosolic and mitochondrial iron accumulation in cultured hippocampal neurons (Fig. 1a, b) and in the hippocampus of developing rats (Fig. 1c, d) and aged mice (Fig. 1d, e).

Deferiprone (DFP) is a low-molecular weight iron chelator, which has been clinically used to treat iron overload, particularly for patients with haemosiderosis. DFP can freely cross the blood-brain barrier and bind iron in multiple subcellular and extracellular locations [23, 24]. To see if DFP would reverse the GA-induced overload, we treated the neurons $30 \mathrm{~min}$ or animals $1 \mathrm{~h}$ before GA exposure. The results showed that the effect of ketamine or sevoflurane on the contents of cytosolic and mitochondrial iron was prevented (Fig. 1). These findings suggested that both intravenous and inhalational GAs could cause iron overload in hippocampal neurons and in the developing and aged brains.

\section{GA disturbs neuronal iron metabolism}

Cells maintain optimal intracellular iron levels by iron regulatory proteins (IRP1 and IRP2), which can posttranscriptionally regulate the expression of a set of genes such as transferrin receptors 1 (TfR1) for iron uptake and ferritin for iron storage. Therefore, we measured the 


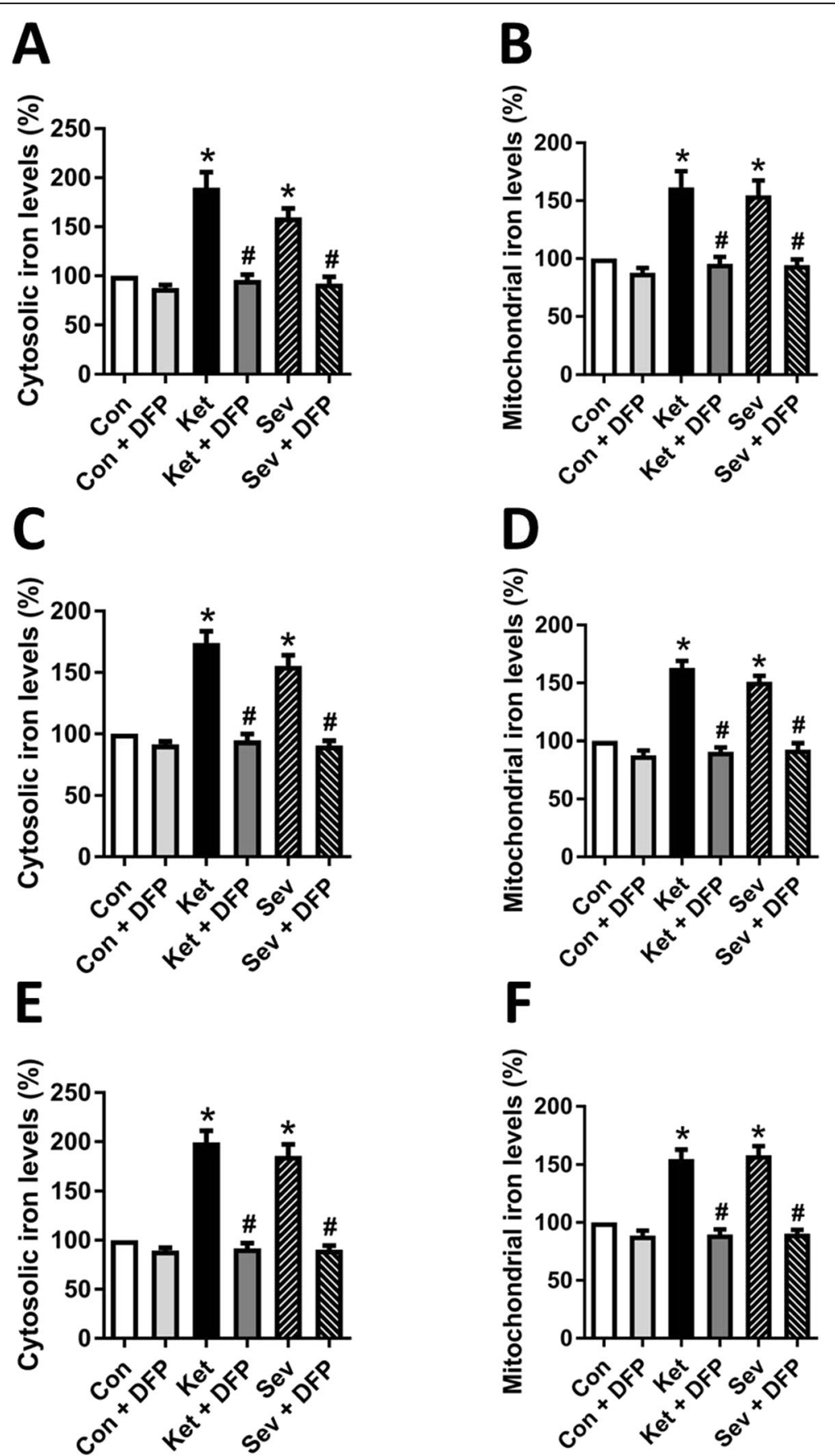

Fig. 1 GA induces hippocampal iron overload. a, b The hippocampal neurons were exposed to ketamine (Ket, $10 \mu \mathrm{M}$ ) or $3 \%$ sevoflurane (Sev) for $6 \mathrm{~h}$ at day 9 in vitro (DIV 9). The cytosolic iron levels (a) and mitochondrial iron levels (b) of hippocampal neurons were measured. $\mathbf{c}$, $\mathbf{d}$ The rat pups at postnatal day (PND) 6 received ketamine $(75 \mathrm{mg} / \mathrm{kg}$ ) intraperitoneally or $3 \%$ sevoflurane $2 \mathrm{~h}$ inhalation daily for three consecutive days. The cytosolic iron levels (c) and mitochondrial iron levels (d) of rat hippocampus were measured. e, $\mathbf{f}$ The 15-month-old mice received ketamine $(75 \mathrm{mg} / \mathrm{kg}$ ) intraperitoneally or $3 \%$ sevoflurane $2 \mathrm{~h}$ inhalation daily for three consecutive days. The cytosolic iron levels (e) and mitochondrial iron levels (f) of the mouse hippocampus were measured. Data are presented as mean \pm SEM $(n=6)$; ${ }^{*} p<0.05$ compared with Con group; ${ }^{\#} p<0.05$ compared with the GA (Ket or Sev) group

expression levels of these proteins after ketamine or sevoflurane exposure. Our results showed that both ketamine and sevoflurane reduced the expression of IRP2 and TfR1 and increased the expression of ferritin in the cultured hippocampal neurons (Fig. 2a) and in the hippocampus of developing rats (Fig. 2b) and aged mice (Fig. 2c), respectively. Moreover, depletion of iron with DFP successfully revered the iron status (Fig. 2). 


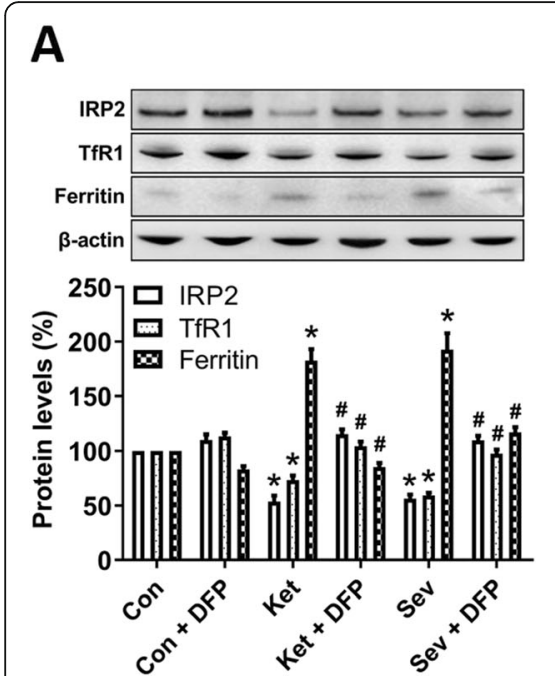

B

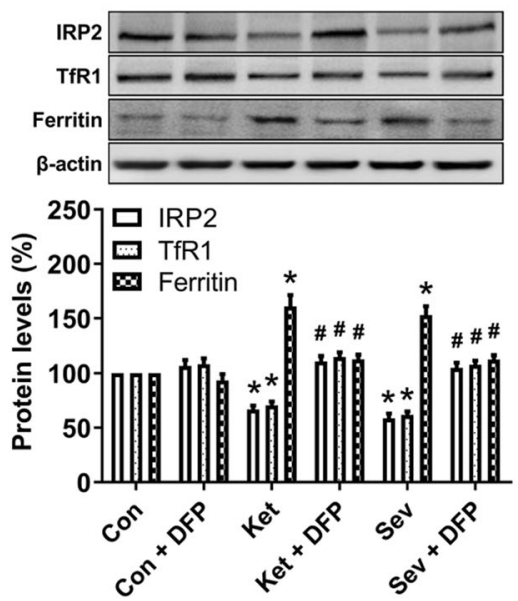

C

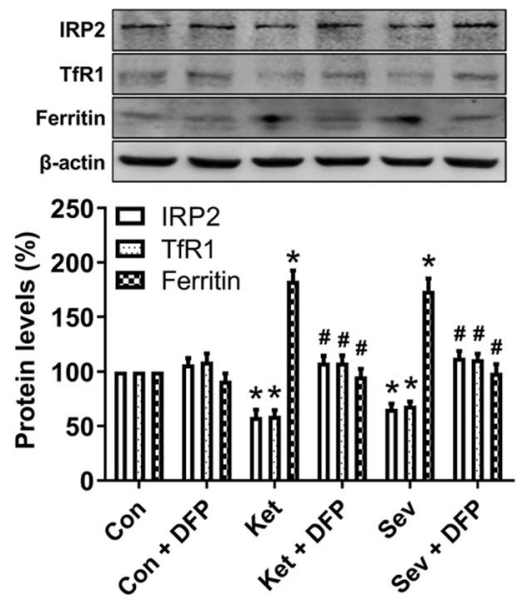

Fig. 2 GA disturbs neuronal iron metabolism. Western blot analysis of iron-related proteins including IRP2 (iron regulatory protein 2 ), TfR1 (an iron uptake protein), and ferritin (an iron storage protein). A representative image set is presented for the cultured hippocampal neurons at DIV 9 (a), the hippocampus of rat pups at PND 8 (b), and the hippocampus of 15-month-old mice (c). The drug treatment is same as in Fig. 1. Data are presented as mean \pm SEM $(n=6) ;{ }^{*} p<0.05$ compared with Con group; ${ }^{*} p<0.05$ compared with the GA (Ket or Sev) group

Mitochondria play a vital role in cellular iron metabolism. These organelles are the major hubs of iron utilization and the sole site of heme synthesis and the major site for biogenesis of iron-sulfur clusters, which are vital for cell functions [25-27]. The mechanisms of mitochondrial iron transport have not been completely revealed. The best known pathway is inward iron transport mediated by mitoferrin (Mfrn), a protein located in the inner mitochondrial membrane $[25,28]$. In this study, we found that the Mfrn1 level was significantly increased in the GA-treated groups when compared to the control group in the cultured hippocampal neurons (Fig. 3a) and in the hippocampus of developing rats (Fig. 3b) and aged mice (Fig. 3c). Taken together, it is demonstrated that both ketamine and sevoflurane intensify the import of iron into cytoplasm and mitochondria, resulting in the accumulation of iron in the cells or tissues.

\section{GA-induced iron overload causes mitochondrial dysfunction}

Neurons are very sensitive to iron overload, of which the toxicity may lead to neuronal mitochondrial dysfunction [25-27]. We have previously demonstrated that mitochondrial dysfunction is involved in the earliest pathogenesis of GA-induced cognitive impairments in developing or aged rodent brain [17, 29]. Whether the GA-induced iron overload elicits mitochondrial dysfunction is not understood. To address this question, we investigated the iron involvement in between GA and mitochondrial dysfunction.

Mitochondria are highly dynamic organelles that continuously divide and fuse through the processes of fission and fusion. First, we measured the protein levels of Drp1 and Mfn2, which are fission and fusion-related proteins, respectively. The result showed that the levels of Drp1 elevated and no significant variation of Mfn2 levels was observed after ketamine or sevoflurane exposure, whereas iron chelation by DFP reversed the expression of Drp1 (Fig. 3a-c), suggesting that iron overload induced by GA exposure led to the mitochondrial fragmentation. Then, we examined the mitochondrial morphology after incubation of the hippocampal neurons with mito-tracker by the confocal microscopy following GA exposure. Consistent with the changes of mitochondrial dynamic proteins, mitochondria became fragmented and condensed after $6 \mathrm{~h}$ of ketamine or sevoflurane treatment (Fig. 3d). Finally, we evaluated the mitochondrial function by determining the levels of mitochondrial permeability transition pore (mPTP) opening, MMP, and ATP production, three important parameters of mitochondrial function-related indexes. The results showed that the opening of mPTP (Fig. 3e) was enhanced, and MMP (Fig. 3f) and ATP production (Fig. 3g) were reduced after GA exposure. However, all the GA-induced effects were prevented by pretreatment with DFP as shown in Fig. 3.

Collectively, these data support that mitochondrial dynamics and function are impaired by ketamine or sevoflurane exposure and iron chelation may ameliorate the injury.

\section{$\mathrm{GA}$ triggers iron-mediated ferroptosis}

Ferroptosis is an important cell death pathway for a number of diseases, including cancer and neurological disorders 

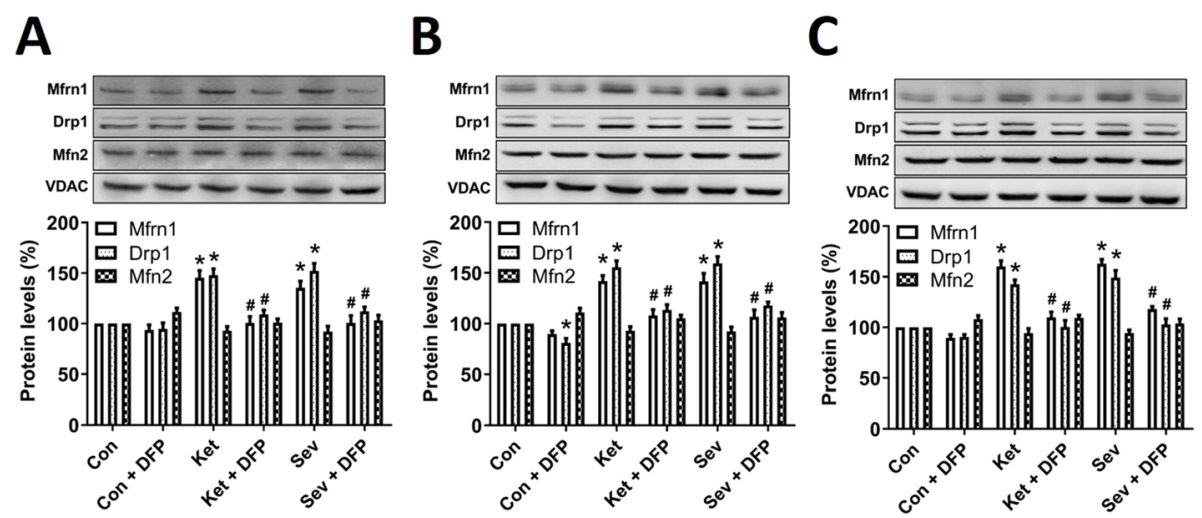

D

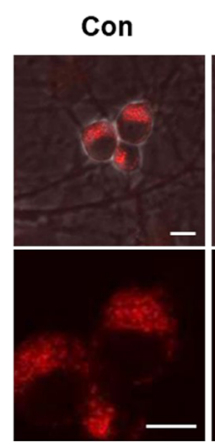

$$
\text { DFP }
$$
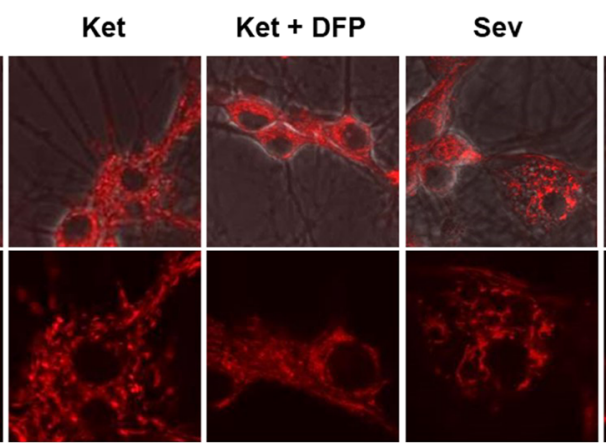

Sev + DFP

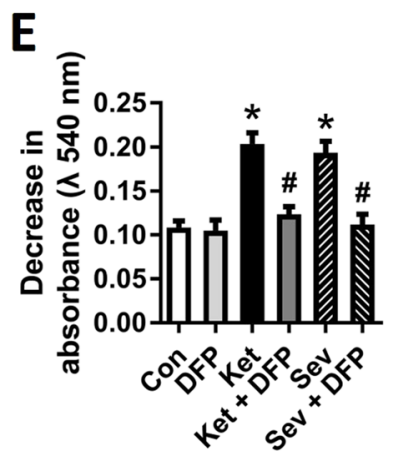

$\mathbf{F}$

G
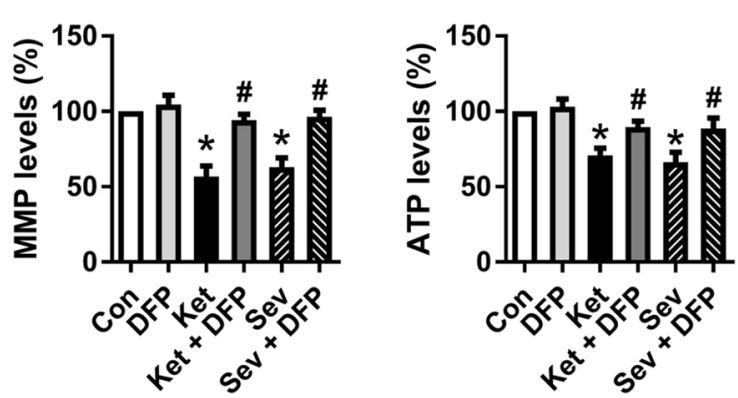

Fig. 3 GA causes neuronal mitochondrial dysfunction. a-c Western blot analysis of mitochondrial iron import protein Mfrn1, mitochondrial fission protein Drp1, and fusion protein Mfn1. A representative image set is presented. Tissues or neurons were lysed and mitochondria were isolated from rat hippocampal neurons (a), rat-pup hippocampus (b), and aged-mouse hippocampus (c). d Mitochondrial morphology. After GA treatment for $6 \mathrm{~h}$, the hippocampal neurons were stained with MitoTracker Red at $37^{\circ} \mathrm{C}$ for $30 \mathrm{~min}$ and observed under the confocal microscopy at $\times 600$ magnification. Scale bar $10 \mu \mathrm{m}$. e Mitochondrial permeability transition pore (mPTP) opening, determined by mitochondrial swelling assay kit with freshly isolated mitochondria. $\mathbf{f}$ Mitochondrial membrane potential (MMP) levels. g ATP production. Data are presented as mean \pm SEM $(n=6) ;{ }^{*} p$ $<0.05$ compared with Con group; ${ }^{\#}<0.05$ compared with the GA (Ket or Sev) group

[15]. It can be prevented by iron chelators such as DFP, deferoxamine (DFO), ciclopirox (CPX), and 2,2-bipyridyl (2, 2-BP) [30, 31]. The selective ferroptosis inhibitor, ferrostatin-1, is able to mitigate neonatal isofluraneinduced neuronal death [16]. Here, we hypothesize that ferroptosis may play an important role in both ketamineand sevoflurane-induced neurotoxicity.

Although the characteristics of ferroptosis have not been perfectly understood, the key biomarkers include iron dependence and the stress levels of lipid peroxidation [32]. Here, we showed that GA treatment induced cells to exhibit condensed and ruptured mitochondria (Fig. 3d), which also belongs to the morphological characteristics of ferroptotic cells [33]. Not only the cell morphology (Fig. 4a), viability (Fig. 4b), and cytotoxicity (Fig. 4c) but also other biomarkers (Fig. 4dg) all matched the phenotypes which represents the process of ferroptosis, and they were all rescued by iron chelation. For instance, the general reactive oxygen species (ROS, Fig. 4d), the final lipid peroxidation product 


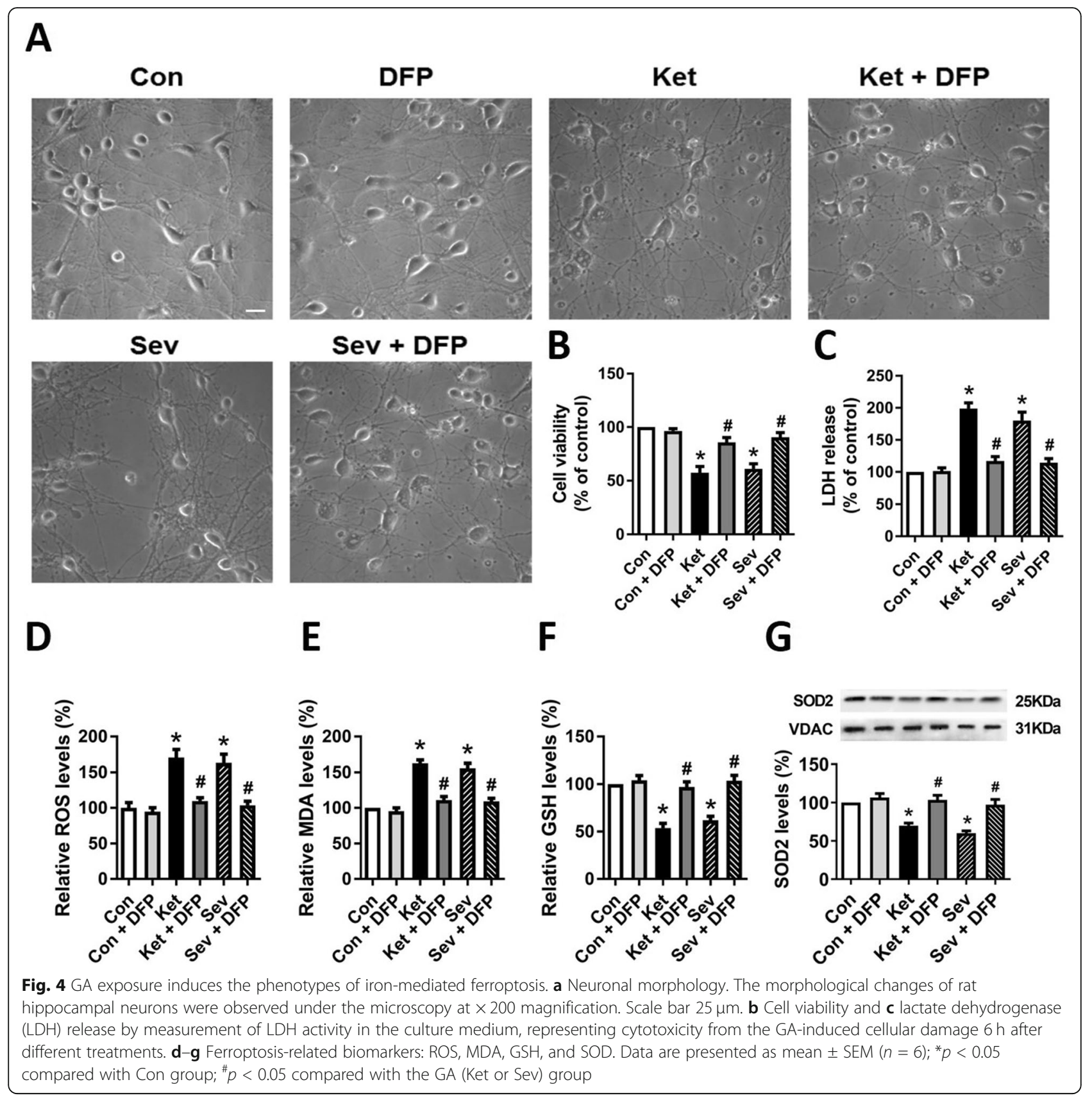

MDA (Fig. 4e), reduced GSH (Fig. 4f) content, and even the scavenger SOD2 (Fig. 4g) altered after GA exposure and reversed after DFP treatment. These results support the notion that ferroptosis is derived from irondependent neurotoxicity after GA exposure.

\section{Chelating neurotoxic iron ameliorates GA-induced cognitive deficits}

To estimate the extent to which GA-induced cognitive outcomes were attributed to iron overload, the adolescent rats were engaged in MWM tests on the indicated days after GA exposure with DFP pretreatment. The
MWM tests showed long escape latency in the spatial training tests (Fig. 5a) and decreased target quadrant time and crossing platform times (Fig. $5 \mathrm{~b}-\mathrm{d}$ ) in probe trial in adolescent rats after exposure to either ketamine or sevoflurane. Interestingly, the swim speed was not found significantly changed (Fig. 5e). For aged mice, fear conditioning tests were performed and showed, comparing to the control, a decreased percentage of freezing time in the 24-h context test (Fig. 5f, g), which reflects the impairment of hippocampus-dependent memory, after ketamine or sevoflurane treatment. Beyond our expectation, DFP pretreatment remarkably improved the 


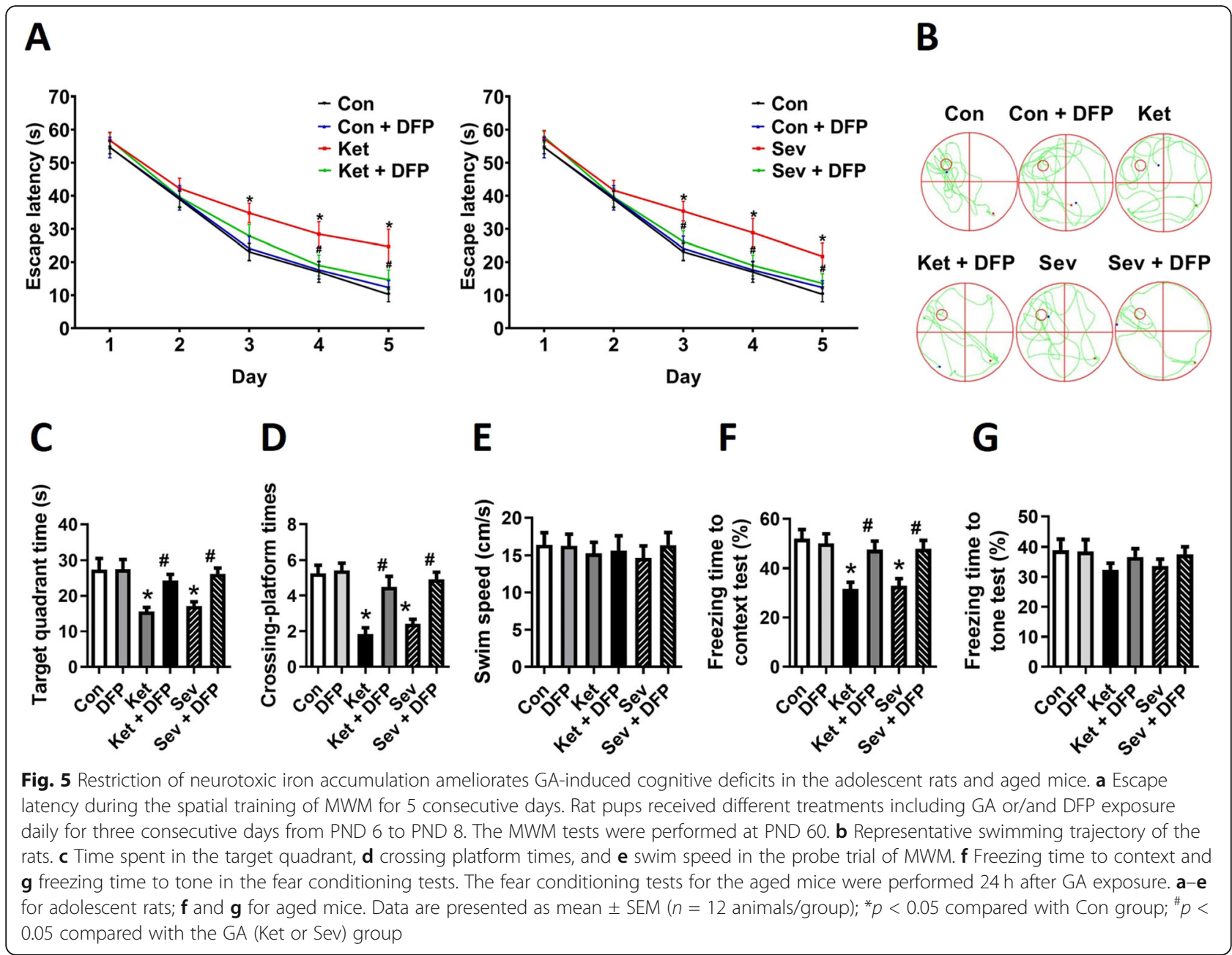

performance in the behavioural tests (Fig. 5). These results indicate that chelating neurotoxic iron with DFP ameliorated GA-induced cognitive deficits and suggest that iron restriction might provide a preventative effect for paediatric patients undertaking GA and might have a therapeutic effect for POCD treatment.

\section{NMDAR-mediated iron transport pathway is involved in GA-induced iron overload}

Based on the above results, we further explored the molecular mechanism underlying GA-mediated iron overload in the brain. NMDAR is one of the two main targets to the GAs. Previous experiments revealed that ketamine or sevoflurane treatment upregulates the expression of NMDAR subunits (compensatory regulation as a consequence of continued or prolonged NMDAR blockade) [34-36]. Moreover, NMDAR upregulation leads to the activation of RASD1 (also called as Dexras1), a novel GTPase, which interacts with iron importer DMT1 and enhances DMT1-mediated iron uptake and iron releasing from lysosome [8, 37]. Here, we found increased expression of NMDAR1 (NR1) and NMDAR2A (NR2A), as well as RASD1 and DMT1, after $6 \mathrm{~h}$ ketamine or sevoflurane treatment (Fig. 6a). To reveal whether GA-induced iron accumulation could be via the enhanced iron uptake, a DMT1-specific inhibitor (DMT1i) $[19,20]$ was utilized. Intriguingly, DMT1i pretreatment before ketamine or sevoflurane exposure significantly suppressed the expression of all the tested components, NR1, NR2, RASD1, and even DMT1 (Fig. 6a), and iron contents in cytosol and mitochondria were also maintained evenly (Fig. 6b) in rat hippocampal neuronal culture. Amazingly, without any swim speed change (Fig. 6c), DMT1i ameliorated the GA-induced cognitive impairments in vivo in all the tested assays including escape latency (Fig. 6d), target quadrant time (Fig. 6e) and crossing platform times (Fig. 6f) in MWM tests of the rats, and freezing time (Fig. 6f) in fear conditioning test of the aged mice. The freezing time to tone test (Fig. 6h) did not show the difference after DMT1i treatment. Our data, overall, confirmed the effect of GAinduced iron neurotoxicity and demonstrated that 


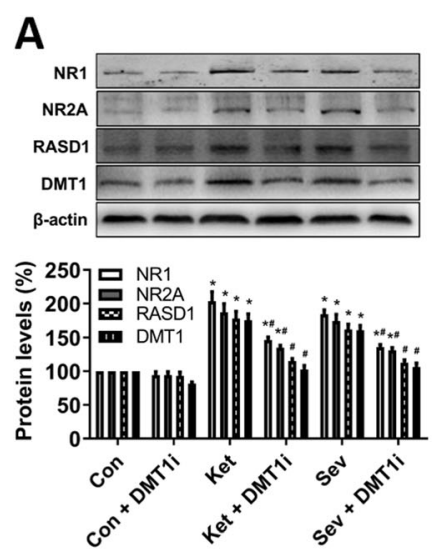

B

C
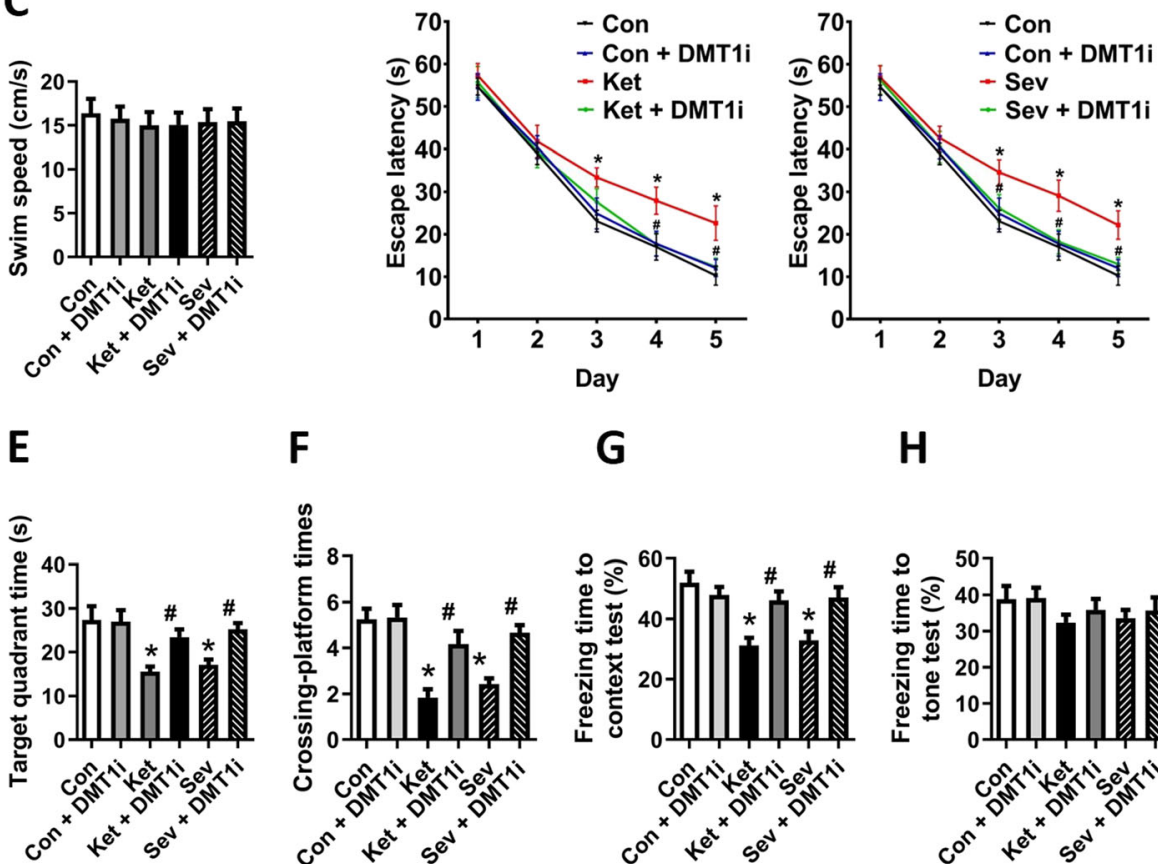

$\mathbf{F}$

G

H
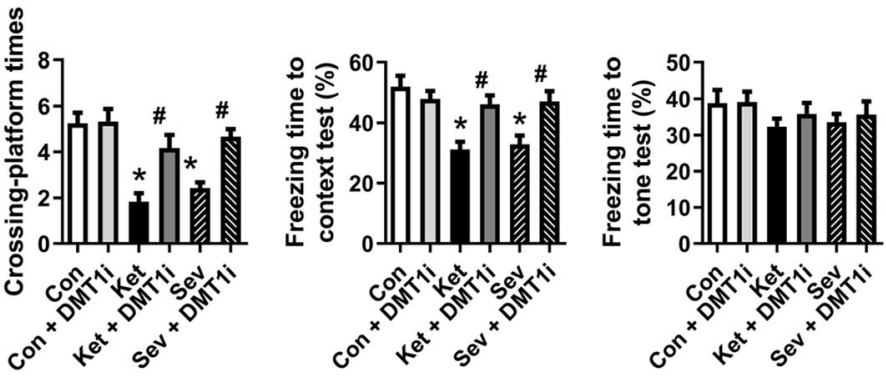

Fig. 6 GA-induced activation of NMDAR-RASD1-mediated DMT1 iron uptake signalling pathway. a To inhibit NMDAR-RASD1-mediated DMT1 iron uptake signalling pathway, DMT1i was added to the culture medium 30 min before GA exposure. Protein levels of NMDAR, RASD1, and DMT1 were determined by Western blot. A representative image set is presented. $\mathbf{b}$ The cytosolic and mitochondrial iron levels of hippocampal neurons. c Swim speed, $\mathbf{d}$ escape latency, e time spent in the target quadrant, and $\mathbf{f}$ crossing platform times in MWM tests. $\mathbf{g}$ Freezing time to context and $\mathbf{h}$ freezing time to tone in the fear conditioning tests. $\mathbf{a}$ and $\mathbf{b}$ for hippocampal neurons; $\mathbf{c}-\mathbf{f}$ for adolescent rats; $\mathbf{g}$ and $\mathbf{h}$ for aged mice. Data are presented as mean \pm SEM ( $n=12$ animals/group); ${ }^{*} p<0.05$ compared with Con group; ${ }^{*} p<0.05$ compared with the GA (Ket or Sev) group

blockade of iron uptake would provide a feedback effect on NMDAR-mediated signalling to protect neurons from damage.

\section{Discussion}

This study provides the first integrative evidence that intravenous and inhalational GAs, such as ketamine and sevoflurane, may induce iron overload in the hippocampus of developing and aged brains to be involved in the cognitive deficits, likely, through neuronal ferroptosis. Our study shed new light on understanding the mechanism of GAs to generate neurotoxicity, at least partly, through modulation of iron metabolism in the brain.

The developing or aged brain has several significant differences from the adult brain that provide a physiological basis for enhanced resistance to insults, including exposure to perioperative stressors [4,5]. Recent studies have demonstrated that excess iron may be detrimental for neural development, behavioural and cognitive functioning, particularly, at the extremes of age $[10,13,14]$. In this study, we found that both ketamine and 
sevoflurane disrupted iron homeostasis by affecting the expression of proteins involved in iron uptake further to induce cytosolic and mitochondrial iron accumulation in the cultured hippocampal neurons and in the hippocampus of developing rats and aged mice. Interestingly, pretreatment with DFP successfully ameliorated the turbulence of iron metabolism and attenuated the ketamine- or sevoflurane-induced neuronal toxicity. Previous studies have revealed that surgical trauma induces iron accumulation in the hippocampus and that iron chelator DFO, whose mode of action is still under investigation, protects against neuroinflammation and cognitive impairments in an aged rodent model of POCD [38, 39]. However, the blood-brain barrier is thought to be relatively impermeable to DFO [40]. Unlike DFO, DFP, a small positively charged molecule (only $139 \mathrm{Da}$ ), is neutral in the circulation and, whether free or bound to iron, readily penetrates cells [41]. Therefore, we used DFP to chelate the accumulated cellular iron in brain or ex vivo neurons, which is in line with the clinical firstline treatment for removing and/or preventing iron accumulation in the brain [42].

Mitochondria are important organelles in all cell types, particularly in the neurons. Mitochondrial dysfunction has been previously shown to link to the earliest pathogenesis of GA-induced cognitive impairments in developing or aged mammalian brain $[17,29]$. On the one hand, we confirmed our previous findings including the mitochondrial dysfunction after GA exposure [43]; on the other hand, we observed that pretreatment with DFP prior to GA exposure significantly attenuated GAinduced effects on mitochondrial function and dynamics. This means that GA-induced impairment of mitochondria is directly associated with iron accumulation, which is consistent with the previous studies in other ironoverload models [44]. Therefore, we can draw a rough conclusion that iron toxicity is involved in the mechanism underlying GA-induced mitochondrial dysfunction.

Ferroptosis is a relatively new form of regulated cell death. Though still more to be unfolded, it involves iron dependence and lethal lipid-based ROS generation. Emerging data suggest that ferroptosis is one, if not the main, driver of cell death in cancer and neurodegenerative diseases like Parkinson's disease, Alzheimer's disease, and Friedreich's ataxia $[15,45]$. In the present study, our results showed that ketamine or sevoflurane initiated neuronal death, presenting the combination of a few ferroptotic biomarkers including iron dependence, increased lipid peroxidation, and decreased GSH content [32]. Among them, it is very attractive that iron restriction by iron chelation with DFP or blockage of iron uptake with DMT1i not only abolishes GA-induced ferroptosis but also resists GA-induced neurotoxicity and cognitive deficits. Both ways to limit the iron availability are to restrict NTBI in a short time frame, suggesting that the iron dependence of ferroptosis is rather NTBI- than other sources of iron dependence. Recently, it has been demonstrated that increase of DMT1 expression, not increased TfR1 or decreased Fpn1, may be mainly responsible for aging or disease-dependent increase of iron in brain [46], which may share the same reason as GA-induced iron overload in this study. The previous studies have demonstrated that anaesthesia has a significant impact on phosphorylation of tau and on neuropathology of cognitive decline in Alzheimer's disease [47]. A recent study has addressed that iron deposition leads to hyperphosphorylation of tau and impairs cognition in mic e[48]. Thus, hyperphosphorylation of tau, as a result of iron overload, is supposed to be another mechanism of GA-induced cognitive deficits.

Evidence proved that NMDAR upregulation could promote iron overload and iron-induced neurotoxicity by enhancing RASD1-DMT1-mediated iron uptake [8] and iron releasing from the lysosome [9]. In the present study, we provided direct evidence that ketamine or sevoflurane exposure caused an upregulation of NMDAR subunits, which might be a compensatory upregulation of NMDAR subunits [34-36]. Accompanying with that, both ketamine and sevoflurane induced upregulation of RASD1, which could form a ternary complex with PAP7 and DMT1 and thus enhance the ability of NMDAR to activate DMT1 for iron uptake $[8,37]$. The activation was inhibited by administration of DMT1 inhibitor DMT1i in this study to prevent iron accumulation in neurons, which attenuated iron-induced neurotoxicity. Interestingly, DMT1i treatment reduced the protein levels of NR1, NR2A, RASD1, and DMT1. One possible mechanism is that DMT1i ameliorates GA-induced iron overload and consequently provides a feedback to counteract the GA-induced compensatory upregulation of NMDAR. At the end, it causes the downregulation of NR1, NR2A, and RASD1. The similar feedback regulation was also observed previously [9]. Therefore, it is possible that modulation of iron level is an effective strategy for prevention from NMDARinduced neurotoxicity.

\section{Conclusion}

This is the first time, at least to our knowledge, to reveal that iron overload is involved in GA-induced neurotoxicity and cognitive deficits. This work suggests a new strategy that local depletion of iron or restriction of iron influx might be beneficial for the treatment of GA or iron overload-induced neurodevelopmental or neurodegenerative toxicity.

\section{Abbreviations}

GA: General anaesthesia; GAs: General anaesthetics; POCD: Postoperative cognitive dysfunction; NMDA: N-Methyl-D-aspartate; GABA: Gamma- 
aminobutyric acid; DMT1: Divalent metal transporter 1; NTBI: Non-transferrinbound iron; DFP: Deferiprone; IRP: Iron regulatory proteins; TfR1: Transferrin receptors 1; Mfrn: Mitoferrin; mPTP: Mitochondrial permeability transition pore; MMP: Mitochondrial membrane potential

\section{Acknowledgements}

We really appreciate all the members in Dr. Li's and Dr. Yang's laboratories for their valuable comments to the current study.

\section{Authors' contributions}

$J W, S Y$, and $K L$ conceived and designed the experiments. JW, JJY, CY, HL, HZ, and SY performed the experiments and analysed the data. JW, SY, and KL wrote the manuscript. All authors discussed, edited, and approved the final version of the manuscript.

\section{Funding}

This study was supported by the National Natural Science Foundation of China (grants 31871201, 81700423, 81873526, and 81901944).

\section{Availability of data and materials}

All data generated in this study are included in this manuscript.

\section{Ethics approval and consent to participate}

All experimental procedures and protocols were reviewed and approved by the Animal Investigation Ethics Committee of Nanjing University and were performed in accordance with the Guidelines for the Care and Use of Laboratory Animals from the National Institutes of Health, USA.

\section{Consent for publication}

Not applicable.

\section{Competing interests}

The authors declare that they have no competing interests.

\section{Author details}

${ }^{1}$ Jiangsu Key Laboratory of Molecular Medicine, Medical School of Nanjing University, 22 Hankou Road, Nanjing 210093, China. ${ }^{2}$ Department of Anesthesiology, The first Affiliated Hospital of Zhengzhou University, Zhengzhou 450000, China. ${ }^{3}$ Department of Vascular Surgery, Renji Hospital, School of Medicine, Shanghai Jiaotong University, Shanghai 200127, China.

\section{Received: 10 December 2019 Accepted: 17 March 2020}

\section{Published online: 11 April 2020}

\section{References}

1. Disma N, O'Leary JD, Loepke AW, Brambrink AM, Becke K, Clausen NG, De Graaff JC, Liu F, Hansen TG, McCann ME, et al. Anesthesia and the developing brain: a way forward for laboratory and clinical research. Paediatr Anaesth. 2018;28:758-63.

2. Mutch WAC, El-Gabalawy RM, Graham MR. Postoperative delirium, learning, and anesthetic neurotoxicity: some perspectives and directions. Front Neurol. 2018;9:177.

3. Walters JL, Paule MG. Review of preclinical studies on pediatric general anesthesia-induced developmental neurotoxicity. Neurotoxicol Teratol. 2017; $60: 2-23$.

4. Jungwirth B, Zieglgansberger W, Kochs E, Rammes G. Anesthesia and postoperative cognitive dysfunction (POCD). Mini-Rev Med Chem. 2009;9: 1568-79.

5. Hudson $\mathrm{AE}$, Hemmings $\mathrm{HC}$ Jr. Are anaesthetics toxic to the brain? Br J Anaesth. 2011;107:30-7.

6. Belelli D, Pistis M, Peters JA, Lambert JJ. General anaesthetic action at transmitter-gated inhibitory amino acid receptors. Trends Pharmacol Sci. 1999;20:496-502.

7. Franks NP, Honore E. The TREK K2P channels and their role in general anaesthesia and neuroprotection. Trends Pharmacol Sci. 2004;25:601-8.

8. Cheah JH, Kim SF, Hester LD, Clancy KW, Patterson SE 3rd, Papadopoulos V, Snyder SH. NMDA receptor-nitric oxide transmission mediates neuronal iron homeostasis via the GTPase Dexras1. Neuron. 2006;51:431-40.

9. White RS, Bhattacharya AK, Chen Y, Byrd M, McMullen MF, Siegel SJ, Carlson GC, Kim SF. Lysosomal iron modulates NMDA receptor-mediated excitation via small GTPase, Dexras1. Mol Brain. 2016;9:38.
10. Singh N, Haldar S, Tripathi AK, Horback K, Wong J, Sharma D, Beserra A, Suda S, Anbalagan C, Dev S, et al. Brain iron homeostasis: from molecular mechanisms to clinical significance and therapeutic opportunities. Antioxid Redox Signal. 2014;20:1324-63.

11. Codazzi F, Pelizzoni I, Zacchetti D, Grohovaz F. Iron entry in neurons and astrocytes: a link with synaptic activity. Front Mol Neurosci. 2015;8:18.

12. Knutson MD. Non-transferrin-bound iron transporters. Free Radic Biol Med. 2019:133:101-11.

13. Amin $S B$, Myers $G$, Wang H. Association between neonatal iron overload and early human brain development in premature infants. Early Hum Dev. 2012;88:583-7.

14. Ward RJ, Zucca FA, Duyn JH, Crichton RR, Zecca L. The role of iron in brain ageing and neurodegenerative disorders. Lancet Neurol. 2014;13:1045-60.

15. Li J, Cao F, Yin HL, Huang ZJ, Lin ZT, Mao N, Sun B, Wang G. Ferroptosis: past, present and future. Cell Death Dis. 2020;11:88.

16. Xia Y, Sun X, Luo Y, Stary CM. Ferroptosis Contributes to isoflurane neurotoxicity. Front Mol Neurosci. 2018:11:486.

17. Wu J, Hao S, Sun XR, Zhang H, Li H, Zhao H, Ji MH, Yang JJ, Li K. Elamipretide (SS-31) ameliorates isoflurane-induced long-term impairments of mitochondrial morphogenesis and cognition in developing rats. Front Cell Neurosci. 2017;11:119.

18. Zhang H, Sun XR, Wang J, Zhang ZZ, Zhao HT, Li HH, Ji MH, Li KY, Yang JJ. Reactive oxygen species-mediated loss of phenotype of parvalbumin interneurons contributes to long-term cognitive impairments after repeated neonatal ketamine exposures. Neurotox Res. 2016;30:593-605.

19. Cadieux JA, Zhang Z, Mattice M, Brownlie-Cutts A, Fu J, Ratkay LG, Kwan R, Thompson J, Sanghara J, Zhong J, Goldberg YP. Synthesis and biological evaluation of substituted pyrazoles as blockers of divalent metal transporter 1 (DMT1). Bioorg Med Chem Lett. 2012;22:90-5.

20. Xue X, Ramakrishnan SK, Weisz K, Triner D, Xie L, Attili D, Pant A, Gyorffy B Zhan M, Carter-Su C, et al. Iron uptake via DMT1 integrates cell cycle with JAK-STAT3 signaling to promote colorectal tumorigenesis. Cell Metab. 2016; 24:447-61.

21. Seibenhener ML, Wooten MW. Isolation and culture of hippocampal neurons from prenatal mice. J Vis Exp. 2012.

22. Li H, Zhao H, Hao S, Shang L, WU J, Song C, Meyron-Holtz EG, Qiao T, Li K. Iron regulatory protein deficiency compromises mitochondrial function in murine embryonic fibroblasts. Sci Rep. 2018;8:5118.

23. Fredenburg AM, Sethi RK, Allen DD, Yokel RA. The pharmacokinetics and blood-brain barrier permeation of the chelators 1,2 dimethly-, 1,2 diethyl-, and 1-[ethan-1'ol]-2-methyl-3-hydroxypyridin-4-one in the rat. Toxicology. 1996;108:191-9.

24. Sohn YS, Breuer W, Munnich A, Cabantchik ZI. Redistribution of accumulated cell iron: a modality of chelation with therapeutic implications. Blood. 2008;111:1690-9.

25. Paul BT, Manz DH, Torti FM, Torti SV. Mitochondria and Iron: current questions. Expert Rev Hematol. 2017;10:65-79.

26. Pelizzoni I, Macco R, Morini MF, Zacchetti D, Grohovaz F, Codazzi F. Iron handling in hippocampal neurons: activity-dependent iron entry and mitochondria-mediated neurotoxicity. Aging Cell. 2011;10:172-83.

27. Seo AY, Xu J, Servais S, Hofer T, Marzetti E, Wohlgemuth SE, Knutson MD, Chung HY, Leeuwenburgh C. Mitochondrial iron accumulation with age and functional consequences. Aging Cell. 2008:7:706-16.

28. Huang J, Chen S, Hu L, Niu H, Sun Q, Li W, Tan G, Li J, Jin L, Lyu J, Zhou H. Mitoferrin-1 is involved in the progression of Alzheimer's disease through targeting mitochondrial iron metabolism in a Caenorhabditis elegans model of Alzheimer's disease. Neuroscience. 2018;385:90-101.

29. Wu J, Zhang M, Li H, Sun X, Hao S, Ji M, Yang J, Li K. BDNF pathway is involved in the protective effects of SS-31 on isoflurane-induced cognitive deficits in aging mice. Behav Brain Res. 2016;305:115-21.

30. Masaldan S, Clatworthy SAS, Gamell C, Meggyesy PM, Rigopoulos AT, Haupt S, Haupt Y, Denoyer D, Adlard PA, Bush Al, Cater MA. Iron accumulation in senescent cells is coupled with impaired ferritinophagy and inhibition of ferroptosis. Redox Biol. 2018;14:100-15.

31. Dixon SJ, Lemberg KM, Lamprecht MR, Skouta R, Zaitsev EM, Gleason CE, Patel DN, Bauer AJ, Cantley AM, Yang WS, et al. Ferroptosis: an irondependent form of nonapoptotic cell death. Cell. 2012;149:1060-72.

32. Yang WS, Stockwell BR. Ferroptosis: death by lipid peroxidation. Trends Cell Biol. 2016;26:165-76.

33. Cao JY, Dixon SJ. Mechanisms of ferroptosis. Cell Mol Life Sci. 2016;73:2195209. 
34. Liu F, Patterson TA, Sadovova N, Zhang X, Liu S, Zou X, Hanig JP, Paule MG, Slikker W Jr, Wang C. Ketamine-induced neuronal damage and altered Nmethyl-D-aspartate receptor function in rat primary forebrain culture. Toxicol Sci. 2013;131:548-57.

35. Sinner B, Friedrich $\mathrm{O}$, Lindner R, Bundscherer A, Graf BM. Long-term NMDA receptor inhibition affects NMDA receptor expression and alters glutamatergic activity in developing rat hippocampal neurons. Toxicology. 2015;333:147-55

36. Haseneder R, Starker L, Berkmann J, Kellermann K, Jungwirth B, Blobner M, Eder M, Kochs E, Rammes G. Sevoflurane anesthesia improves cognitive performance in mice, but does not influence in vitro long-term potentation in hippocampus CA1 stratum radiatum. PLoS One. 2013;8:e64732.

37. Chen Y, Mathias L, Falero-Perez JM, Kim SF. PKA-mediated phosphorylation of Dexras1 suppresses iron trafficking by inhibiting S-nitrosylation. FEBS Lett. 2015:589:3212-9.

38. Li Y, Pan K, Chen L, Ning JL, Li X, Yang T, Terrando N, Gu J, Tao G. Deferoxamine regulates neuroinflammation and iron homeostasis in a mouse model of postoperative cognitive dysfunction. J Neuroinflammation. 2016:13:268.

39. An LN, Yue Y, Guo WZ, Miao YL, Mi WD, Zhang H, Lei ZL, Han SJ, Dong L. Surgical trauma induces iron accumulation and oxidative stress in a rodent model of postoperative cognitive dysfunction. Biol Trace Elem Res. 2013; 151:277-83.

40. Shachar DB, Kahana N, Kampel V, Warshawsky A, Youdim MB. Neuroprotection by a novel brain permeable iron chelator, VK-28, against 6hydroxydopamine lession in rats. Neuropharmacology. 2004:46:254-63.

41. Cappellini MD, Pattoneri P. Oral iron chelators. Annu Rev Med. 2009;60:2538

42. Kontoghiorghe CN, Kontoghiorghes GJ. Efficacy and safety of iron-chelation therapy with deferoxamine, deferiprone, and deferasirox for the treatment of iron-loaded patients with non-transfusion-dependent thalassemia syndromes. Drug Des Devel Ther. 2016;10:465-81.

43. Wu J, Li H, Sun X, Zhang H, Hao S, Ji M, Yang J, Li K. A mitochondriontargeted antioxidant ameliorates isoflurane-induced cognitive deficits in aging mice. PLoS One. 2015;10:e0138256.

44. Sripetchwandee J, Wongjaikam S, Krintratun W, Chattipakorn N, Chattipakorn SC. A combination of an iron chelator with an antioxidant effectively diminishes the dendritic loss, tau-hyperphosphorylation, amyloids-beta accumulation and brain mitochondrial dynamic disruption in rats with chronic iron-overload. Neuroscience. 2016;332:191-202.

45. Murphy MP. Metabolic control of ferroptosis in cancer. Nat Cell Biol. 2018; 20:1104-5.

46. Lu LN, Qian ZM, Wu KC, Yung WH, Ke Y. Expression of iron transporters and pathological hallmarks of Parkinson's and Alzheimer's diseases in the brain of young, adult, and aged rats. Mol Neurobiol. 2017;54:5213-24.

47. Whittington RA, Bretteville A, Dickler MF, Planel E. Anesthesia and tau pathology. Prog Neuro-Psychopharmacol Biol Psychiatry. 2013;47:147-55.

48. Wan W, Cao L, Kalionis B, Murthi P, Xia S, Guan Y. Iron deposition leads to hyperphosphorylation of tau and disruption of insulin signaling. Front Neurol. 2019:10:607.

\section{Publisher's Note}

Springer Nature remains neutral with regard to jurisdictional claims in published maps and institutional affiliations.

Ready to submit your research? Choose BMC and benefit from:

- fast, convenient online submission

- thorough peer review by experienced researchers in your field

- rapid publication on acceptance

- support for research data, including large and complex data types

- gold Open Access which fosters wider collaboration and increased citations

- maximum visibility for your research: over $100 \mathrm{M}$ website views per year

At BMC, research is always in progress.

Learn more biomedcentral.com/submissions 